\title{
Risk factors for neonatal intensive care unit admission in Amman, Jordan
}

\author{
C.E. Quinn, ${ }^{7}$ P. Sivasubramaniam, ${ }^{7}$ M. Blevins, ${ }^{2,3}$ A. Al Hajajra, ${ }^{5}$ A. Taleb Znait, ${ }^{5}$ N. Khuri-Bulos, ${ }^{5,6}$ S. Faouri ${ }^{5}$ and N. Halasa ${ }^{2,4,7}$
}

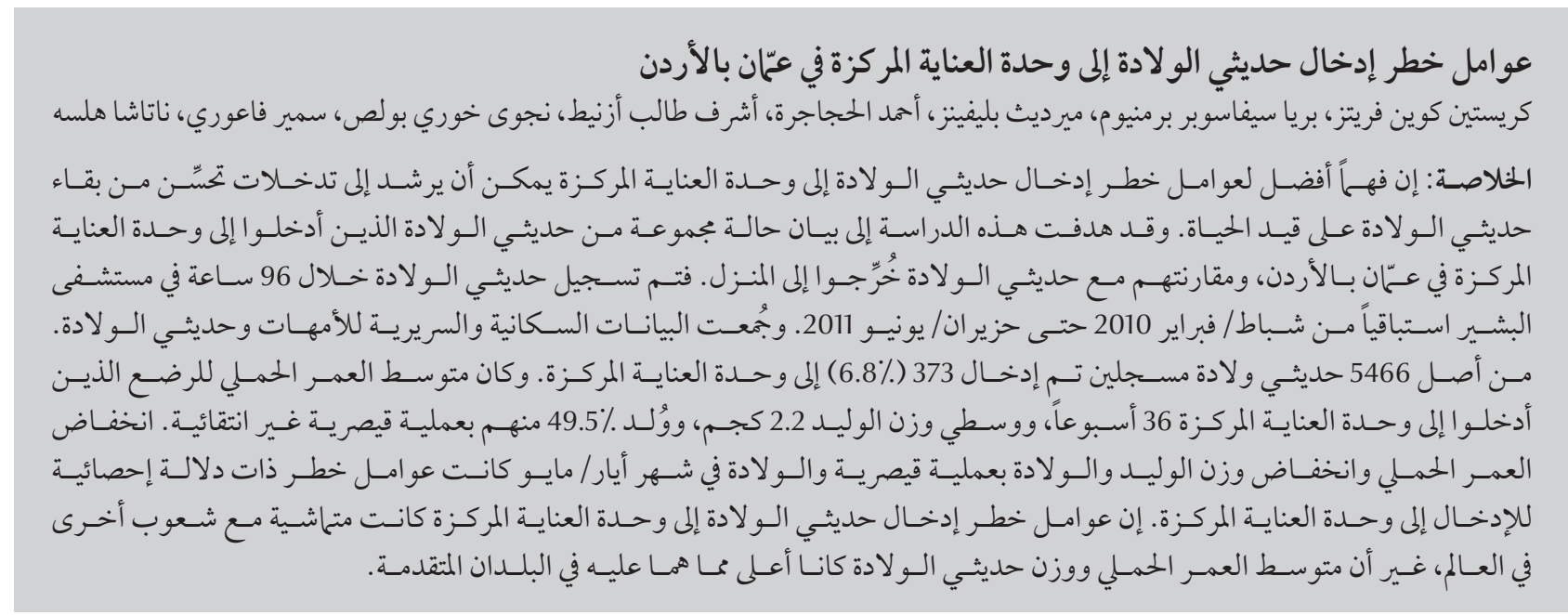

ABSTRACT A better understanding of risk factors for neonatal intensive care unit (NICU) admission can inform interventions to improve neonatal survival. This study aimed to describe a population of newborns admitted to a NICU in Amman, Jordan, and compare them with newborns discharged to home. Newborns born within 96 hours at Al-Bashir Hospital were enrolled from February 2010 to June 2011. Demographic and clinical data were collected for mothers and newborns. Of 5466 enrolled neonates, 373 (6.8\%) were admitted to the NICU. The median gestational age of NICU infants was 36 weeks, median birth weight was $2.2 \mathrm{~kg}$ and $49.5 \%$ were delivered by non-elective caesarean section. Lower gestational age, lower birth weight, delivery by caesarean section and birth in the month of May were statistically significant risk factors for NICU admission. Risk factors for NICU admission were consistent with other populations worldwide; however, median gestational age and birth weight were higher than in developed countries.

\section{Facteurs de risque pour l'admission en unité de soins intensifs néonatals à Amman (Jordanie)}

RÉSUMÉ Une meilleure compréhension des facteurs de risque pour l'admission en unité de soin intensifs néonatals permet d'orienter les interventions en vue d'améliorer la survie des nouveau-nés. La présente étude avait pour objectif de décrire une population de nouveau-nés admis en unité de soins intensifs néonatals à Amman (Jordanie) et de mener une étude comparative avec des nouveau-nés rentrés à domicile. Les nouveau-nés dont la naissance est survenue dans un laps de temps de 96 heures après leur entrée à l'hôpital Al-Bashir ont participé à l'étude entre février 2010 et juin 2011. Des données démographiques et cliniques ont été collectées pour les mères et les nouveau-nés. Sur 5466 nouveau-nés, 373 (6,8\%) ont été admis en unité de soins intensifs néonatals. L'âge gestationnel médian des enfants admis dans l'unité était de 36 semaines, le poids médian à la naissance était de 2,2 kg et 49,5\% d'entre eux étaient nés par césarienne non élective. Un âge gestationnel plus bas, un poids à la naissance plus faible, une naissance par césarienne et la naissance au cours du mois de mai constituaient des facteurs de risque statistiquement significatifs pour une admission dans l'unité. Ces facteurs de risque coïncidaient avec ceux d'autres populations dans le monde, mais l'âge gestationnel et le poids à la naissance médians étaient plus élevés que dans les pays développés.

${ }^{7}$ Undergraduate Medical Education; ${ }^{2}$ Vanderbilt Institute for Global Health, Nashville, Tennessee, United States of America. ${ }^{3}$ Department of Biostatistics; ${ }^{4}$ Department of Paediatrics, Vanderbilt University School of Medicine, Nashville, Tennessee, United States of America (Correspondence to C.E. Quinn: cristin.e.quinn@vanderbilt.edu). ${ }^{5}$ Al-Basheer Government Hospital, Amman, Jordan. ${ }^{6}$ Division of Infectious Disease, Jordan University Hospital, Amman, Jordan. ' Vanderbilt University Medical Centre, Nashville, Tennessee, United States of America. Received: 02/09/14; accepted: 10/12/15 


\section{Introduction}

Neonatal intensive care has become a cornerstone for treatment of premature infants worldwide. In the United States, for example, wide access to advances in neonatal care such as surfactant therapy and antenatal corticosteroids has resulted in an increase in survival of neonates admitted to NICUs across the country (1). These technological advances have led to an average cohort that would have likely been considered non-viable just a few decades ago. Neonates weighing under $1500 \mathrm{~g}$ have shown a steady increase in survival over the past decade, and neonates born at 25 weeks now have a $75 \%$ survival rate $(2,3)$. In addition to improvements in survival among the most premature neonates, the United States has seen a steady decrease in its overall neonatal mortality rate (deaths under age 28 days per 1000 live births) and the most current estimate is around 4 per 1000 live births (4).

Globally, childhood mortality is of particular interest as the world strives to meet Millennium Development Goal (MDG) 4: reducing child mortality by two-thirds between 1990 and 2015 (5). Currently, $40 \%$ of deaths in children younger than 5 years old occur in the neonatal period, with $99 \%$ of neonatal deaths occurring in low- or middle-income countries (6). This represents a 30-fold higher average daily mortality rate during the neonatal period than the post-neonatal period (7). Complications related to preterm birth accounted for the largest fraction $(14.1 \%)$ of these deaths, followed by intrapartum-related complications (9.4\%) and neonatal sepsis or meningitis $(5.2 \%)(8)$. Though there has been an overall reduction in the under-5 mortality rate (U5MR) by an average of $2.6 \%$ per year, the decrease falls short of the $4.4 \%$ annual decrease needed to reach MDG 4. Additionally, decreases in U5MR have occurred more rapidly in children outside of the neonatal period, so that the neonatal fraction of deaths increased from $38.2 \%$ to $40.3 \%$ of deaths between 2000 and 2010 (8). Thus, interventions focusing on reducing neonatal death, particularly those caused by preterm birth, are crucial to achieving MDG 4.

This trend is similar in Jordan, where the U5MR is 21 per 1000 live births (9). During the last two decades neonatal morbidity has grown to represent a larger proportion of overall infant mortality (10), with prematurity as the leading cause of the USMR (34\%), followed by congenital anomalies (21\%) and birth asphyxia (12\%) $(9,11)$. This calls for a focus on neonatal care to reduce the U5MR and achieve MDG 4. However, neither basic demographic information nor risk factors for admission of infants to NICUs in the Middle East, including Jordan, are well-documented in the current literature. A better understanding of the characteristics of infants requiring care in the NICU can provide direction for interventions that would improve neonatal survival. Therefore, in this study we aimed to describe the demographic and clinical characteristics of mothers and infants admitted to a NICU and to estimate the risk factors for admission to the NICU of Al Bashir Government Hospital, one of three major hospitals serving Amman, Jordan.

\section{Methods}

The study was designed as a retrospective cohort nested within a prospective cohort study. This study was approved by the University of Jordan and the institutional review boards of Vanderbilt University and the Jordanian Ministry of Health at Al Bashir Hospital.

\section{Sample}

This study was part of a larger neonatal cohort study that enrolled neonates born at Al Bashir Government Hospital for baseline assessment of their vitamin D levels. If cohort members were subsequently admitted for respiratory illness, another vitamin D level was drawn at that time (12). Female research assistants approached all mothers on the postpartum ward during daytime hours from Sunday to Thursday. Verbal consent was obtained from mothers to obtain heelpricks for blood from their neonates. Over the period 1 February 2010 to 30 June 2011 all neonates born at the hospital within 96 hours were eligible for inclusion in the study. However, the majority of infants were discharged within 24 hours unless theywere admitted to the NICU.

The medical records of neonates from the prospective cohort who were admitted to the NICU were obtained and reviewed retrospectively to collect additional data about NICU admission and outcomes.

\section{Data collection}

Using a standardized case report questionnaire, mothers in the prospective cohort study were queried in Arabic by a member of the research team, who subsequently recorded the answers in English. The research assistants all received prior training in the protocol for questionnaire administration to ensure consistency. Parents were asked to provide the nationality of the mother and father, child's date of birth, route of delivery, child's birth weight, mother's vitamin D supplementation history, daily number of hours that mother spends outdoors, mother's clothing practice, whether or not the mother smoked during pregnancy (and if so, which of the trimesters), and if the mother was exposed to smoke in her household during pregnancy. The questionnaire also asked for self-reported diagnoses with medical conditions that may be associated with decreased bone health, including hyperparathyroidism, gestational diabetes, rheumatoid arthritis, and diseases requiring corticosteroid treatment. 
For the retrospective data collection an electronic form was used to collect the following variables for every neonate admitted to the NICU: reason for admission, Apgar score, C-reactive protein measurements, duration of stay in the NICU, presence of complications associated with prematurity, early and late infection status, antibiotic administration, surfactant administration and ventilation strategy. Apgar score was considered low if it was $<7$. Maternal risk factors such as gestational diabetes mellitus, premature rupture of membranes, chorioamnionitis and pre-eclampsia were also recorded when documented in the infant's chart. All data were entered into a secured electronic database (Vanderbilt REDCap) (13).

\section{Statistical analysis}

Descriptive statistics were used to summarize the sociodemographic and clinical characteristics of newborns by NICU admission. A multivariable logistic regression model was used to estimate the association of maternal exposure and birth outcomes with NICU admission. Covariates were identified a priori, and they included: baby's sex, gestational age, birth weight and type of delivery; mother's age, education, prenatal care, clothing practice, hours spent outdoors, exposure to tobacco smoke and any vitamin D supplementation; and baby's date of birth (temporal trend). To account for possible non-linear associations, continuous variables were included in the models using restricted cubic splines (14). Missing values of covariates were accounted for using multiple imputation techniques which used predictive mean matching to take random draws from imputation models; 25 imputation data sets were used in the analysis (15). We employed $R$ statistical software, version 2.15.1 (www.r-project. org) for all data analyses. Analysis scripts are available at http://biostat. mc.vanderbilt.edu/ArchivedAnalyses.

\section{Results}

During the 17 -month study period, 19604 babies were born at Al-Bashir Hospital: 2810 non-viable (14.3\%) and 16794 living neonates. Of the living neonates, 3317 (19.8\%) were admitted to the NICU.

\section{Characteristics of prospective cohort sample}

During that same time period, we approached 6057 mothers of neonates and enrolled 5466 neonates (90\%) in our prospective cohort (2697 females and 2769 males) (Figure 1). The neonates in the prospective cohort had a median gestational age of 39 weeks, median birth weight of $3.1 \mathrm{~kg}$ and caesarean section delivery rate of $20 \%$ ( Table 1). All caesarean sections performed were non-elective.

The mothers in the cohort study had a median age of 27 years and $24 \%$ had a history of multivitamin use during pregnancy. The vast majority of women were healthy; the two most common medical conditions reported by mothers were rheumatoid arthritis (2\%) and gestational hypertension (2\%). Although only $7 \%$ of mothers reported smoking during pregnancy, $72 \%$ reported exposure to tobacco smoke.

Of the 5466 neonates in the prospective cohort, 373 (7\%) were admitted to the NICU during the study period (Table 1). Nearly half of these (183/373) were caesarean section deliveries, of which 53 (29\%) were for fetal indications and 130 (71\%) were for maternal indications.

\section{Newborn characteristics by NICU admission}

In the retrospective analysis comparing NICU to non-NICU neonates, NICU neonates had a lower median gestational age (36 weeks versus 39 weeks, $P<0.001)$; lower median birth weight ( $2.2 \mathrm{~kg}$ versus $3.1 \mathrm{~kg}, P<0.001$ ); and were more likely to be delivered by caesarean section (49\% versus 18\%, $P$
$<0.001$ ) (Table 1). Neonates of Palestinian nationality were more likely to be admitted to the NICU compared with neonates of other nationalities (12\% NICU versus 9\% non-NICU, $P$ $=0.018)$. No differences in vitamin $\mathrm{D}$ levels were noted, with a median of 3.5 $\mathrm{ng} / \mathrm{mol}$ in both groups. Certain vitamin supplements were more commonly used during pregnancy by the mothers of NICU neonates than non-NICU neonates: a multivitamin, vitamin $\mathrm{D}$, folic acid, calcium and iron $(P<0.001)$. NICU neonates were also more likely to be born to a mother that had a higher body mass index, history of diabetes, gestational diabetes and pregnancyinduced hypertension than were nonNICU neonates $(P<0.001$, Table 1$)$. Other maternal factors-including age, level of education, clothing practice and location of prenatal care were not significantly different between the two groups.

\section{Multivariable logistic regression analysis of risk factors for NICU admission}

Multivariable logistic regression was employed to estimate neonatal and maternal factors that were independently associated with admission to the NICU (Table 2, Figure 2). Lower gestational age was associated with NICU admission. Compared with a gestational age of 40 weeks, neonates born at 34 or 36 weeks had a higher risk of NICU admission (OR 8.4; 95\% CI: 5.6-12.4 and OR 3.6; 95\% CI: 2.7-4.8 respectively). Neonates born weighing $2.5 \mathrm{~kg}$ had over 5 times higher odds of NICU admission compared with those with a birth weight of $3 \mathrm{~kg}$ (OR 5.39; 95\% CI: 4.44-6.55). Delivery by caesarean section had over twice the odds of NICU admission (OR 2.36; 95\% CI: 1.71-3.23). Babies born in May 2010 and May 2011 were also more likely to be admitted to the NICU (OR 2.43; 95\% CI: 1.45-4.10). No significant associations were detected between NICU admission and maternal factors 


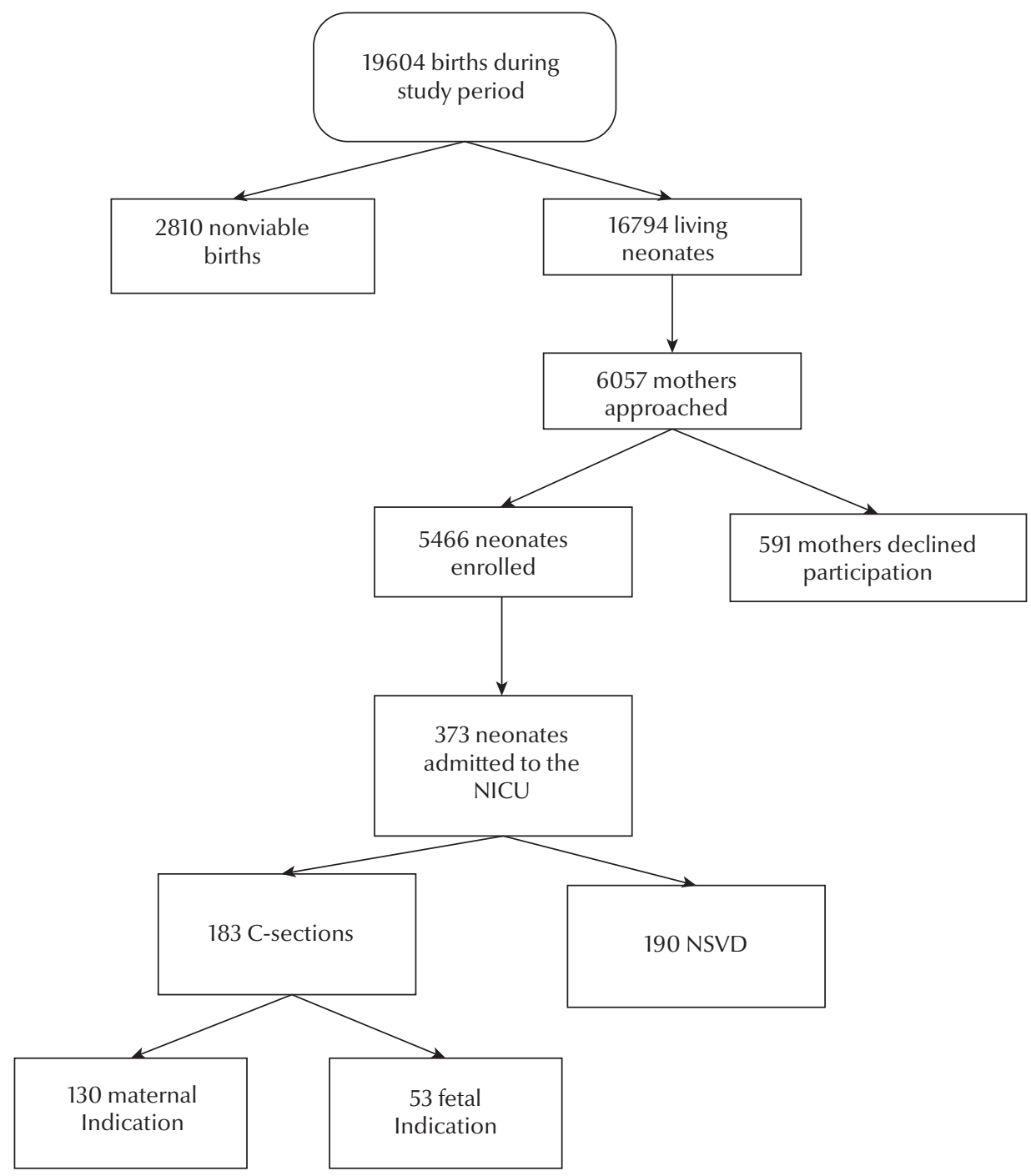

Figure 1 Overview of prospective cohort sample (NICU = neonatal intensive care unit; NSVD = normal spontaneous vaginal delivery)

such as age, education level, location of prenatal care or clothing practice.

\section{NICU patient characteristics and outcomes by delivery type}

To further characterize differences of neonates admitted to the NICU, we compared neonates delivered by vaginal delivery or caesarean section (Table 3). Of the 373 NICU neonates, 318 (85\%) had charts available for retrospective review. Comparing mode of delivery, children born by vaginal delivery had a significantly higher median gestational age [interquartile range (IQR) 35-37 weeks versus $34-37$ weeks, $P=0.016$ ] and higher median birth weight $(2.30 \mathrm{~kg}$ versus $2.25 \mathrm{~kg}, P=0.039)$ than neonates delivered by caesarean section. Additionally, compared with vaginal delivery, caesarean section was associated with the following characteristics: admission for low Apgar score (11\% versus 1\%, $P<0.001$ ), use of nasal cannula (44\% versus $30 \%, P=0.01$ ), use of surfactant ( $12 \%$ versus $4 \%, P=0.029$ ), lower white blood cell count (13.1 versus $13.9 \times$ $\left.10^{9} / \mathrm{L}, P=0.047\right)$, more days in the hospital (4 days versus 3 days, $P=0.002$ ) and more days on antibiotics (4 days versus 3 days, $P=0.002$ ) (Table 3 ). Maternal diagnosis of pre-eclampsia was significantly associated with a caesarean section delivery (18\% versus $2 \%$,
$P<0.001)$. Neonates born by caesarean section had a higher rate of death in the hospital than neonates born vaginally, but this difference was not significant (10\% versus $7 \%, P=0.506)$.

\section{Discussion}

This is one of the first studies describing neonates admitted to a NICU in Amman, Jordan and estimating the associated risk factors for NICU admission. In ourstudy, wefound that neonates admitted to the NICU had lower birth weight and gestational age compared with neonates not admitted to the NICU. 


\begin{tabular}{|c|c|c|c|c|}
\hline Variable & $\begin{array}{c}\text { NICU } \\
(n=373)\end{array}$ & $\begin{array}{l}\text { Non-NICU } \\
(n=5093)\end{array}$ & $\begin{array}{c}\text { Total } \\
(n=5466)\end{array}$ & $P$-value \\
\hline \multicolumn{5}{|l|}{ Sex } \\
\hline Female [no. (\%)] & $165(44)$ & $2532(50)$ & $2697(49)$ & 0.047 \\
\hline \multicolumn{5}{|l|}{ Gestational age } \\
\hline Gestational age (weeks) [median (IQR)] & $36(34-38)$ & $39(38-40)$ & $39(38-40)$ & $<0.001$ \\
\hline Age range (weeks) & $25-43$ & $24-43$ & $24-43$ & \\
\hline \multicolumn{5}{|l|}{ Birth weight } \\
\hline Birth weight (kg) [median (IQR)] & $2.2(1.9-3)$ & $3.1(2.9-3.5)$ & $3.1(2.8-3.5)$ & $<0.001$ \\
\hline Weight range (kg) & $0.5-5.6$ & $1.3-5.0$ & $0.5-5.6$ & \\
\hline Type of delivery [no. (\%)] & & & & $<0.001$ \\
\hline Normal spontaneous vaginal & $190(51)$ & $4162(82)$ & $4352(80)$ & \\
\hline Caesarean section & $183(49)$ & $929(18)$ & $1112(20)$ & \\
\hline \multicolumn{5}{|l|}{ Mother's age } \\
\hline Age (years) [median (IQR)] & $27(23-33)$ & $27(22-32)$ & $27(22-32)$ & 0.154 \\
\hline \multicolumn{5}{|l|}{ Mother's BMI } \\
\hline BMI $\left(\mathrm{kg} / \mathrm{m}^{2}\right)[$ median $(\mathrm{IQR})]$ & $25.0(21.6-28.0)$ & $23.6(21.3-26.6)$ & $23.8(21.4-26.7)$ & $<0.001$ \\
\hline Missing values [no. (\%)] & $176(47)$ & $3330(65)$ & $3506(64)$ & \\
\hline Mother's highest education level [no. (\%)] & & & & 0.347 \\
\hline Primary education & $84(34)$ & $883(33)$ & $967(33)$ & \\
\hline Secondary education & $130(52)$ & $1293(49)$ & $1423(49)$ & \\
\hline College education & $31(12)$ & $370(14)$ & $401(14)$ & \\
\hline No education & $4(2)$ & $92(3)$ & $96(3)$ & \\
\hline Missing values & $124(33)$ & $2455(48)$ & $2579(47)$ & \\
\hline Mother's nationality [no. (\%)] & & & & 0.018 \\
\hline Jordanian & $310(83)$ & $4413(87)$ & $4723(86)$ & \\
\hline Egyptian & $14(4)$ & $140(3)$ & $154(3)$ & \\
\hline Palestinian & $46(12)$ & $446(9)$ & $492(9)$ & \\
\hline Other & $2(1)$ & $94(2)$ & $96(2)$ & \\
\hline Father's nationality [no. (\%)] & & & & 0.158 \\
\hline Jordanian & $315(84)$ & $4485(88)$ & $4800(88)$ & \\
\hline Egyptian & $17(5)$ & $155(3)$ & $172(3)$ & \\
\hline Palestinian & $38(10)$ & $406(8)$ & $444(8)$ & \\
\hline Other & $3(1)$ & $47(1)$ & $50(1)$ & \\
\hline \multicolumn{5}{|l|}{ Tobacco smoke exposure [no. (\%)] } \\
\hline Primary exposure & $28(8)$ & $379(7)$ & $407(7)$ & 1.000 \\
\hline Secondary exposure & $266(71)$ & $3616(71)$ & $3882(71)$ & 0.944 \\
\hline Any exposure & $268(72)$ & $3665(72)$ & $3933(72)$ & 1.000 \\
\hline \multicolumn{5}{|l|}{ Location of prenatal care [no. (\%)] } \\
\hline $\mathrm{MOH}$ & $83(22)$ & $818(16)$ & $901(16)$ & 0.002 \\
\hline UNRWA & $76(20)$ & $1053(21)$ & $1129(21)$ & 0.943 \\
\hline Private sector & $146(39)$ & $1687(33)$ & $1833(34)$ & 0.020 \\
\hline University & $1(<1)$ & $12(<1)$ & $13(<1)$ & 1.000 \\
\hline Other & $3(1)$ & $20(<1)$ & $23(<1)$ & 0.441 \\
\hline None specified & $111(30)$ & $2099(41)$ & $2210(40)$ & \\
\hline \multicolumn{5}{|l|}{ Time spent outdoors } \\
\hline Median time (IQR) (hours/day) & $0.5(\underline{0.5-1.5)}$ & $1.0(0.5-2.0)$ & $1.0(0.5-2.0)$ & 0.568 \\
\hline
\end{tabular}




\begin{tabular}{|c|c|c|c|c|}
\hline Variable & $\begin{array}{c}\text { NICU } \\
(n=373)\end{array}$ & $\begin{array}{l}\text { Non-NICU } \\
(n=5093)\end{array}$ & $\begin{array}{c}\text { Total } \\
(n=5466)\end{array}$ & $P$-value \\
\hline \multicolumn{5}{|l|}{ Use of supplements } \\
\hline Took vitamin D during pregnancy [no. (\%)] & $72(19)$ & $1128(22)$ & $1200(22)$ & 0.224 \\
\hline \multicolumn{5}{|l|}{ Supplements taken [no. (\%)] } \\
\hline Folic acid & $215(58)$ & $2351(46)$ & $2566(47)$ & $<0.001$ \\
\hline Multivitamins & $155(42)$ & $1140(22)$ & $1295(24)$ & $<0.001$ \\
\hline Calcium & $151(40)$ & $1436(28)$ & $1587(29)$ & $<0.001$ \\
\hline Calcium with vitamin D & $14(4)$ & $96(2)$ & $110(2)$ & 0.022 \\
\hline Vitamin D only & $0(0)$ & $6(<1)$ & $6(<1)$ & 1.000 \\
\hline Iron & $225(60)$ & $2309(45)$ & $2534(46)$ & $<0.001$ \\
\hline Unknown vitamin & $2(1)$ & $39(1)$ & $41(1)$ & 0.853 \\
\hline Other & $6(2)$ & $29(1)$ & $35(1)$ & 0.036 \\
\hline Any vitamin D supplementation & $162(43)$ & $1214(24)$ & $1376(25)$ & $<0.001$ \\
\hline $\begin{array}{l}\text { Received any vaccinations during } \\
\text { pregnancy [no. (\%)] }\end{array}$ & $79(21)$ & $1216(24)$ & $1295(24)$ & 0.263 \\
\hline \multicolumn{5}{|l|}{ Mother's medical history [no. (\%)] } \\
\hline Hyperparathyroidism & $0(0)$ & $4(<1)$ & $4(<1)$ & 1.000 \\
\hline Diabetes & $9(2)$ & $2(<1)$ & $11(<1)$ & $<0.001$ \\
\hline Gestational diabetes & $8(2)$ & $16(<1)$ & $24(<1)$ & $<0.001$ \\
\hline Thyroid dysfunction & $3(1)$ & $34(1)$ & $37(1)$ & 1.000 \\
\hline Renal failure & $0(0)$ & $3(<1)$ & $3(<1)$ & 1.000 \\
\hline Heart disease & $1(<1)$ & $9(<1)$ & $10(<1)$ & 1.000 \\
\hline Asthma & $0(0)$ & $38(1)$ & $38(1)$ & 0.177 \\
\hline Rheumatoid arthritis & $15(4)$ & $105(2)$ & $120(2)$ & 0.021 \\
\hline Chronic disease requiring steroids & $1(<1)$ & $6(<1)$ & $7(<1)$ & 0.973 \\
\hline Hypertension & $7(2)$ & $42(1)$ & $49(1)$ & 0.072 \\
\hline $\begin{array}{l}\text { Gestational (pregnancy-induced) } \\
\text { hypertension }\end{array}$ & $21(6)$ & $71(1)$ & $92(2)$ & $<0.001$ \\
\hline \multicolumn{5}{|l|}{ Total newborn vitamin D } \\
\hline Vitamin D level (nmol/L) [median (IQR)] & $3.5(2.4-5.2)$ & $3.5(2.3-5.0)$ & $3.5(2.3-5.0)$ & 0.269 \\
\hline Missing values [no. (\%)] & $39(10)$ & $1517(30)$ & $1556(28)$ & \\
\hline
\end{tabular}

$I R Q=$ interquartile range; $B M I=$ body mass index; $M O H=$ Ministry of Health; UNRWA = United Nations Relief and Works Agency for Palestine Refugees in the Near East.

However, the median gestational age of 36 weeks and birth weight of $2.2 \mathrm{~kg}$ were higher than median birth weight of $2.0 \mathrm{~kg}$ and gestational age of 31-34 weeks among neonates admitted to NICUs in North America and Europe (16-18). The majority of our neonates would be considered late preterm infants (34-36 weeks). A difference in approach to care at the time of delivery may explain our older NICU cohort. For instance, infants that are extremely premature do not receive the same aggressive resuscitation that they would in developed countries and are considered non-viable. During the study period, the majority of newborns with a gestational age $<26$ weeks were categorized as nonviable. This is consistent with the high $14.3 \%$ non-viable birth rate at Al-Bashir Hospital. Therefore, a further understanding of risk factors for premature birth Amman is needed to reduce the rate of non-viable births, since these neonates are less likely to be admitted to the NICU.

Although the morbidity and mortality of late-preterm infants has been found to be lower than for those who are extremely premature, this group has a greater need for NICU admission, higher morbidity associated with organ immaturity and more long-term neurodevelopmental problems when compared with term infants (19-27). A recent study at a military hospital in Amman, Jordan found that $72.7 \%$ of preterm births were late preterm infants and that these infants experienced significantly higher morbidity and hospitalization than did term infants (28). Factors including male sex, maternal 


\begin{tabular}{|c|c|c|c|}
\hline Variable & OR & $95 \% \mathrm{Cl}$ & $P$-value \\
\hline \multicolumn{4}{|l|}{ Newborn characteristics } \\
\hline \multicolumn{4}{|l|}{ Sex } \\
\hline Male & 1.33 & $1.00-1.77$ & 0.052 \\
\hline Gestational age (weeks) & & & $<0.001$ \\
\hline 34 & 8.36 & $5.63-12.4$ & \\
\hline 36 & 3.59 & $2.69-4.77$ & \\
\hline 38 & 1.62 & $1.27-2.07$ & \\
\hline 40 (Ref.) & 1 & & \\
\hline 42 & 0.78 & $0.50-1.23$ & \\
\hline Birth weight (kg) & & & $<0.001$ \\
\hline 2 & 43.5 & $28.3-66.9$ & \\
\hline 2.5 & 5.39 & $4.44-6.55$ & \\
\hline 3 (Ref.) & 1 & & \\
\hline 3.5 & 1.39 & $1.21-1.60$ & \\
\hline Type of delivery & & & $<0.001$ \\
\hline Vaginal (Ref.) & 1 & & \\
\hline Caesarean section & 2.36 & $1.71-3.23$ & \\
\hline Month of birth & & & $<0.001$ \\
\hline May 2010 & 2.43 & $1.45-4.10$ & \\
\hline August 2010 (Ref.) & 1 & & \\
\hline November 2010 & 0.46 & $0.32-0.66$ & \\
\hline February 2011 & 0.74 & $0.44-1.24$ & \\
\hline May 2011 & 2.15 & $1.40-3.30$ & \\
\hline \multicolumn{4}{|l|}{ Maternal characteristics } \\
\hline \multicolumn{4}{|l|}{ Age } \\
\hline (per 5 years) & 1.02 & $0.92-1.14$ & 0.669 \\
\hline Education & & & 0.717 \\
\hline No education & 0.80 & $0.36-1.75$ & \\
\hline Primary education & 1.15 & $0.80-1.66$ & \\
\hline Secondary education (Ref.) & 1 & & \\
\hline College/university & 1.10 & $0.64-1.90$ & \\
\hline \multicolumn{4}{|l|}{ Location of prenatal care } \\
\hline Any $\mathrm{MOH}$ clinic & 1.53 & $0.94-2.49$ & 0.090 \\
\hline Any UNRWA clinic & 1.35 & $0.85-2.14$ & 0.199 \\
\hline Any private sector clinic & 1.17 & $0.74-1.83$ & 0.501 \\
\hline Clothing practice & & & 0.221 \\
\hline Head scarf only (Ref.) & 1 & & \\
\hline European dress & 3.37 & $0.73-15.6$ & \\
\hline Fully covered & 1.21 & $0.78-1.87$ & \\
\hline Time spent outdoors (hours/day) & 0.98 & $0.85-1.13$ & 0.761 \\
\hline \multicolumn{4}{|l|}{ Tobacco smoke exposure } \\
\hline Primary exposure & 0.68 & $0.39-1.18$ & 0.172 \\
\hline Secondary exposure & 0.90 & $0.65-1.24$ & 0.511 \\
\hline \multicolumn{4}{|l|}{ Use of supplements } \\
\hline Any vitamin D supplementation & 1.63 & $1.15-2.32$ & 0.007 \\
\hline
\end{tabular}

$O R=$ odds ratio; $C l=$ confidence interval; Ref $=$ reference group.

$\mathrm{MOH}=$ Ministry of Health; UNRWA = United Nations Relief and Works Agency for Palestine Refugees in the Near East. 
Points

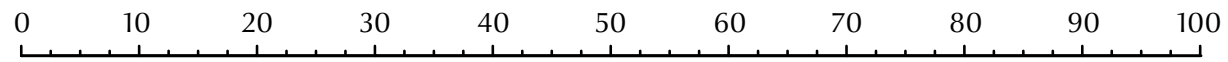

Sex

$$
\text { Female }
$$

Gestational age (weeks)

Birth weight $(\mathrm{kg})$

Delivery

Maternal age (years)

Mother's education

Any $\mathrm{MOH}$ prenatal care

Any UNRWA prenatal care

Any private sector prenatal care

Mother's clothing practice

Hours mother spends outdoors per day

Primary smoking exposure

Secondary smoking exposure

Any vitamin D supplementation

Date of birth

Total Points

Risk of NICU Admission

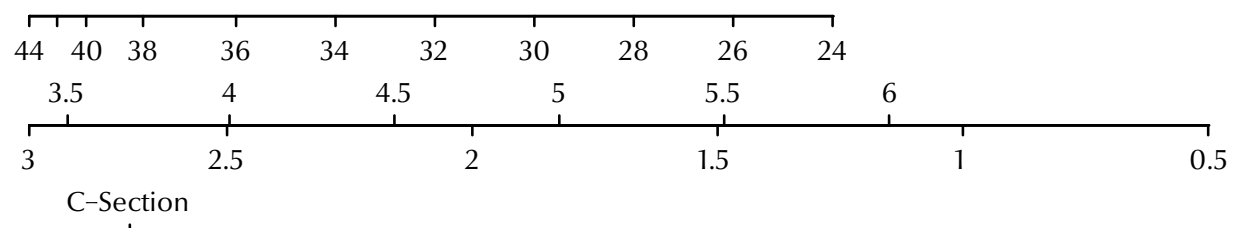

$$
\text { NSVD }
$$

10

Secondary education

$$
\text { rי }
$$

No education

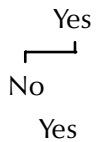

Yes

No

Yes

$r$

No

Fully covered

scarf only

Head scarf only 


\begin{tabular}{|c|c|c|c|c|}
\hline Variable & $\begin{array}{l}\text { Vaginal } \\
(n=162)\end{array}$ & $\begin{array}{c}\text { Caesarean } \\
(n=156)\end{array}$ & $\begin{array}{c}\text { Total } \\
(n=318)\end{array}$ & $P$-value \\
\hline Female sex & $72(44)$ & $81(52)$ & $153(48)$ & 0.222 \\
\hline \multicolumn{5}{|l|}{ Gestational age } \\
\hline Gestational age (weeks) [median (IRQ)] & $36(35-37)$ & $\begin{array}{c}36 \\
(34-37)\end{array}$ & $36(34-37)$ & 0.016 \\
\hline Missing values [no. (\%)] & $2(1)$ & $2(1)$ & $4(1)$ & - \\
\hline \multicolumn{5}{|l|}{ Birth weight } \\
\hline Birth weight (kg) [median (IRQ)] & $2.3(2 .-3)$ & $2.3(1.8-2.8)$ & $\begin{array}{c}2.3 \\
(1.9-2.9)\end{array}$ & 0.039 \\
\hline Missing values [no. (\%)] & $0(0)$ & $1(1)$ & $\begin{array}{c}1 \\
<1\end{array}$ & - \\
\hline Weight at 5 days (kg) [median (IRQ)] & $2.11(1.79-2.83)$ & $1.90(1.58-2.43)$ & $2.00(1.73-2.60)$ & 0.045 \\
\hline Missing values [no. (\%)] & $110(68)$ & $101(65)$ & $211(66)$ & - \\
\hline Days in hospital [median (IRQ)] & $3(1-6)$ & $4(2-9)$ & $3(1-7)$ & 0.002 \\
\hline \multicolumn{5}{|l|}{ Reason(s) for admission [no. (\%)] } \\
\hline Sepsis & $15(9)$ & $16(10)$ & $31(10)$ & 0.912 \\
\hline Respiratory distress syndrome & $105(65)$ & $107(69)$ & $212(67)$ & 0.552 \\
\hline Neonatal pneumonia & $1(1)$ & $0(0)$ & $1(<1)$ & 1.000 \\
\hline Prematurity & $75(46)$ & $89(57)$ & $164(52)$ & 0.071 \\
\hline Heart disease & $3(2)$ & $3(2)$ & $6(2)$ & 1.000 \\
\hline Congenital malformation & $12(7)$ & $6(4)$ & $18(6)$ & 0.258 \\
\hline Overweight infant & $8(5)$ & $9(6)$ & $17(5)$ & 0.936 \\
\hline Low birth weight infant & $22(14)$ & $22(14)$ & $44(14)$ & 1.000 \\
\hline Jaundice & $8(5)$ & $8(5)$ & $16(5)$ & 1.000 \\
\hline Low Apgar score & $2(1)$ & $17(11)$ & $19(6)$ & $<0.001$ \\
\hline Hypoglycaemia & $3(2)$ & $1(1)$ & $4(1)$ & 0.642 \\
\hline Neonatal asphyxia & $4(2)$ & $10(6)$ & $14(4)$ & 0.150 \\
\hline Other & $55(34)$ & $44(28)$ & $99(31)$ & 0.325 \\
\hline Death & $12(7)$ & $15(10)$ & $27(8)$ & - \\
\hline \multicolumn{5}{|l|}{ Apgar scores } \\
\hline Apgar score at $1 \mathrm{~min}$. [median (IRQ)] & $7(5-7)$ & $6(5-7)$ & $7(5-7)$ & 0.339 \\
\hline Missing values [no. (\%)] & $131(81)$ & $10(6)$ & $141(44)$ & - \\
\hline Apgar score at 5 min. [median (IRQ)] & $8(7-8)$ & $8(6-8)$ & $8(7-8)$ & 0.149 \\
\hline Missing values [no. (\%)] & $107(66)$ & $10(6)$ & $117(37)$ & - \\
\hline \multicolumn{5}{|l|}{ Supplement use by mother } \\
\hline Vitamin D (nmol/L) [median (IRQ)] & $3.5(2.5-5.2)$ & $3.5(2.5-4.9)$ & $3.5(2.5-5.0)$ & 0.945 \\
\hline C-reactive protein ever positive [no. (\%)] & & & & 1.000 \\
\hline Missing & $17(10)$ & $12(8)$ & $29(9)$ & - \\
\hline Negative & $133(92)$ & $132(92)$ & $265(92)$ & - \\
\hline Positive & $12(8)$ & $12(8)$ & $24(8)$ & - \\
\hline \multicolumn{5}{|l|}{ White blood cell counts } \\
\hline 1st value $\left(\times 10^{9} / \mathrm{L}\right)[$ median (IRQ)] & $13.9(10.3-18.4)$ & $13.1(9.2-16.8)$ & $13.2(9.9-17.3)$ & 0.047 \\
\hline Missing values [no. (\%)] & $8(5)$ & $0(0)$ & $8(3)$ & - \\
\hline \multicolumn{5}{|l|}{ NICU treatment } \\
\hline Surfactant [no. (\%)] & $7(4)$ & $18(12)$ & $25(8)$ & 0.029 \\
\hline Antibiotics (days) [median (IRQ)] & $3(2-6)$ & $4(3-8)$ & $4(2-7)$ & 0.002 \\
\hline Mechanical ventilation [no. (\%)] & $12(7)$ & $17(11)$ & $29(9)$ & 0.376 \\
\hline Mechanical ventilation (days) [median (IRQ)] & $2(2-3)$ & $4(1-9)$ & $3(1-6)$ & - \\
\hline Nasal cannula [no. (\%)] & $48(30)$ & $69(44)$ & $117(37)$ & 0.010 \\
\hline Nasal cannula (days) [median IRQ)] & $1(1-2)$ & $1(1-3)$ & $1(1-2)$ & - \\
\hline Oxyhood [no. (\%)] & $97(60)$ & 9460 & $191(60)$ & 1.000 \\
\hline Oxyhood (days) [median (IRQ)] & $1(1-2)$ & $1(1-2)$ & $1(1-2)$ & - \\
\hline Incubator [no. (\%)] & $56(35)$ & $52(33)$ & $108(34)$ & 0.909 \\
\hline Incubator (days) [median (IRQ)] & $1(1-2)$ & $2(1-2)$ & $1(1-2)$ & - \\
\hline \multicolumn{5}{|l|}{ Mother's complications [no. (\%)] } \\
\hline Gestational diabetes mellitus & $7(4)$ & $15(10)$ & $22(7)$ & 0.101 \\
\hline Premature rupture of membranes & $11(7)$ & $11(7)$ & $22(7)$ & 1.000 \\
\hline Pre-eclampsia & $3(2)$ & $28(18)$ & $31(10)$ & $<0.001$ \\
\hline
\end{tabular}

$I R Q=$ interquartile range. 
associated with NICU admission (3034). The physiological immaturity associated with young gestational age puts these infants at higher risk of respiratory distress and in greater need for support. Even among neonates delivered by caesarean section, gestational age remains a stronger predictor of NICU admission than rupture of membranes or trial of labour before delivery (35). Maternal factors found to predict NICU admission in other studies include age, race (16), body mass index (36), premature rupture of membranes, antepartum haemorrhage and medical disorders during pregnancy $(32,33)$. Brown et al. suggested that placental ischaemia and endocrine abnormalities associated with these conditions work as biological determinants of preterm birth that act through and with gestational age to produce poor outcomes (34). In our cohort, maternal age and ethnicity were not risk factors for NICU admission.

Interestingly, birth in the month of May was also associated with higher odds of admission to the NICU. No increase in the rate of caesarean sections was noted during this time period, making this unlikely to be the explanation. It is not known why birth in May is a risk factor and therefore this issue merits additional investigation to identify a cause that can be targeted to decrease NICU admission.

In our cohort of NICU admissions, there was a significantly higher caesarean section rate among the neonates admitted to the NICU compared with non-NICU neonates. Our caesarean section rate of $49 \%$ for neonates admitted to the NICU is similar to a study in the United States that found a 50\% caesarean section rate in their NICU cohort (24). In our study, median gestational age and birth weight were lower in neonates delivered by caesarean section compared to those born by vaginal delivery. This weight difference persisted 5 days after birth. In addition, neonates born by caesarean section required more intensive treatment, such as use of surfactant, oxygen by nasal cannula and longer courses of antibiotics than neonates born by vaginal delivery. This finding is consistent with Tita et al., who found caesarean section prior to 39 weeks to be associated with an increased risk of adverse neonatal outcomes such as respiratory complications and admission to the NICU (37). However, their study looked at elective caesarean section, while all of the caesarean sections in our study were non-elective. It is currently unclear whether this is the result of an obstetric practice that is quicker to proceed to caesarean section with any sign of distress or because our cohort is composed of higher risk pregnancies. Another Jordanian study concluded that an over-diagnosis of fetal distress and dystocia was one cause of the unnecessarily high caesarean section rate at King Hussein Medical Centre in Amman (38). These data suggest that working to decrease the number of caesarean sections may be an effective way to decrease admission to the NICU and the associated morbidities. Investigators in China implemented a 6-year programme aiming to decrease the number of caesarean sections, through educational sessions for the entire obstetric care staff, removal of financial incentives for performing caesarean sections, daily review of the indications for each case and improvements in monitoring technology. These interventions resulted in a decrease in the primary caesarean section rate by an annual average of $20 \%$, with a significantly lower rate in the post-intervention time period than the pre-intervention period (39). Therefore, further investigation of the indications for caesarean section in Jordan is needed. If it is found that inappropriate indications are used, an effort similar to the Chinese investigators' could be implemented to help reduce unnecessary caesarean sections and the associated neonatal complications.

There were limitations of our study. The most important was that we were unable to obtain complete information on every neonate born during the study period. Some of the mothers we approached to join the prospective cohort chose not to participate, and there were neonates unaccompanied by their mothers who we were unable to enrol. We expect that our study estimate for NICU admission of $6.8 \%$ is an underestimate, given that the NICU admission rate for the hospital was much higher at $19.8 \%$. This implies that mothers with infants in the NICU were less likely to enrol in our study. If refusals among this population were random, then our assessment of risk factors would be unbiased. If mothers with sicker babies refused, then our results may be biased towards the null as our study NICU population would be more like the general population. If mothers with sicker babies were more likely to participate, then our results would be biased away from the null as our study NICU population would be much different from the general population. We believe that if a bias exists, it would exist towards the null. However, it is possible that the highest risk neonates were not included in our study. Of note, the NICU was undergoing renovations between May 2010 and July 2011, which decreased the overall birth census during this time period.

Other limitations of our study were that we were unable to obtain the medical record of every neonate that was admitted to the NICU during our study period. The surveys used in prospective data collection relied on maternal self-reporting, making them subject to recall and response bias. Finally, the hospital's use of paper charts rather than electronic medical records resulted in missing data in some of the charts that were retrospectively reviewed. However, the major strength of the study is the inclusion of a large neonatal cohort with both maternal and newborn data.

This study represents the first effort in the Middle East to describe a large cohort of neonates admitted to the NICU and to estimate risk factors 
for admission. Our results indicate that the majority of neonates in the Al-Bashir Hospital NICU are late preterm infants. Gestational age, birth weight, caesarean section and birth in the month of May were the most influential risk factors for admission to the NICU. Additionally, there was a much higher rate of caesarean section births among the NICU neonates, and these neonates required more intensive care while in the NICU. Thus, further investigation into risks for preterm birth and why births in May was associated with NICU admission are needed. Additionally, addressing obstetric practices to reduce the number of preterm infants delivered by caesarean section may be an important step in reducing neonatal morbidity and mortality in Jordan. These data serve as a starting point and the study highlights the need for a targeted prospective study in the Arab world to understand risk factors for admission to the NICU.

Funding: None

Competing interests: None declared.

\section{References}

1. Bettegowda V, Lackritz E, Petrini J. Epidemiologic trends in perinatal data. Chapter 3. In: Toward improving the outcome of pregnancy III. White Plains (NY): March of Dimes Foundation; 2010 (http://www.marchofdimes.org/materials/toward-improving-the-outcome-of-pregnancy-iii.pdf, accessed 10 January 2016).

2. Tatad AM, Frayer WW. Trends in the NICU: a review of 25 years' experience. Am J Perinatol. 2003 Nov;20(8):441-6. PMID:14703592

3. NICHD Neonatal Research Network (NRN): extremely preterm birth outcome data [Internet]. Bethesda (MD): National Institute of Child Health and Human Development, Neonatal Research Network; 2012 (https://www.nichd.nih.gov/about/ org/der/branches/ppb/programs/epbo/Pages/epbo_case. aspx, accessed 10 January 2016).

4. MacDorman MF, Hoyert DL, Mathews TJ. Recent declines in infant mortality in the United States, 2005-2011. NCHS Data Brief No. 120. Atlanta (GA): United States Department of Health and Human Services, Centers for Disease Control and Prevention; 2013 (http://www.cdc.gov/nchs/data/databriefs/db120.pdf, accessed 10 January 2016).

5. Millennium Development Goals report 2010. New York: United Nations; 2010 (http://www.un.org/millenniumgoals/ reports.shtml, accessed 10 January 2016).

6. Lawn JE, Wilczynska-Ketende K, Cousens SN. Estimating the causes of 4 million neonatal deaths in the year 2000. Int J Epidemiol. 2006 Jun;35(3):706-18. PMID:16556647

7. Lawn JE, Cousens S, Zupan J; Lancet Neonatal Survival Steering Team. 4 million neonatal deaths: when? Where? Why? Lancet. 2005 Mar 5-11;365(9462):891-900. PMID:15752534

8. Liu L, Johnson HL, Cousens S, Perin J, Scott S, Lawn JE, et al.; Child Health Epidemiology Reference Group of WHO and UNICEF. Global, regional, and national causes of child mortality: an updated systematic analysis for 2010 with time trends since 2000. Lancet. 2012 Jun 9;379(9832):2151-61. PMID:22579125

9. Jordan: WHO statistical profile [Internet]. Geneva: World Health Organization; 2013 (http://www.who.int/gho/countries/jor.pdf, accessed 10 January 2016).

10. Country cooperation strategy for WHO and Jordan 20082013. Cairo: World Health Organization Regional Office for the Eastern Mediterranean; 2009 (http://applications.emro. who.int/docs/CCS_Jordan_2010_EN_14473.pdf, accessed 10 January 2016).

11. Khoury SA, Mas'ad DF. Causes of infant mortality in Jordan. Saudi Med J. 2002 Apr;23(4):432-5. PMID:11953770

12. Khuri-Bulos N, Lang RD, Blevins M, Kudyba K, Lawrence L, Davidson $M$, et al. Vitamin D deficiency among newborns in Amman, Jordan. Glob J Health Sci. 2014 Jan;6(1):162-71. PMID:24373276
13. Harris PA, Taylor R, Thielke R, Payne J, Gonzalez N, Conde JG. Research electronic data capture (REDCap) - a metadata-driven methodology and workflow process for providing translational research informatics support. J Biomed Inform. 2009 Apr;42(2):377-81. PMID:18929686

14. Harrell F. Regression modeling strategies: with applications to linear models, logistic regression, and survival analysis. New York: Springer; 2001.

15. R: a language and environment for statistical computing. Vienna, R Foundation for Statistical Computing; 2012 (http:// www.gbif.org/resource/81287, accessed 10 January 2016).

16. Osterman M, Martin JA, Matthews T, Hamilton BE. Expanded data from the new birth certificate, 2008. National Vital Statistics Reports. 2011;59(7):1-28.

17. Shah P, Yoon E, Chan P. Annual report 2013. Toronto (ON): Canadian Neonatal Network (http://www.canadianneonatalnetwork.org/Portal/LinkClick.aspx?fileticket=IreR0871sjA\%3 D\&tabid=39, accessed 10 January 2016).

18. Van Reempts P, Gortner L, Milligan D, Cuttini M, Petrou S, Agostino R, et al.; MOSAIC Research Group. Characteristics of neonatal units that care for very preterm infants in Europe: results from the MOSAIC study. Pediatrics. 2007 Oct;120(4):e815-25. PMID:17908739

19. Niven GR, Harding JE. Another outcome of neonatal intensive care: first year mortality and hospital morbidity. J Paediatr Child Health. 1995 Apr;31(2):137-42. PMID:7794616

20. Shapiro-Mendoza CK, Tomashek KM, Kotelchuck M, Barfield W, Nannini A, Weiss J, et al. Effect of late-preterm birth and maternal medical conditions on newborn morbidity risk. Pediatrics. 2008 Feb;121(2):e223-32. PMID:18245397

21. Wang ML, Dorer DJ, Fleming MP, Catlin EA. Clinical outcomes of near-term infants. Pediatrics. 2004 Aug;114(2):372-6. PMID:15286219

22. Petrou S, Mehta Z, Hockley C, Cook-Mozaffari P, Henderson J, Goldacre M. The impact of preterm birth on hospital inpatient admissions and costs during the first 5 years of life. Pediatrics. 2003 Dec;112(6 Pt 1):1290-7. PMID:14654599

23. Melamed N, Klinger G, Tenenbaum-Gavish K, Herscovici T, Linder N, Hod M, et al. Short term neonatal outcome in lowrisk spontaneous, singleton, late preterm deliveries. Obstet Gynecol. 2009 Aug;114(2 Pt 1):253-60. PMID:19622985

24. Mally PV, Hendricks-Muñoz KD, Bailey S. Incidence and etiology of late preterm admissions to the neonatal intensive care unit and its associated respiratory morbidities when compared to term infants. Am J Perinatol. 2013 May;30(5):425-31. PMID:23096053

25. Tsai ML, Lien R, Chiang MC, Hsu JF, Fu RH, Chu SM, et al. Prevalence and morbidity of late preterm infants: current status in a medical center of Northern Taiwan. Pediatr Neonatol. 2012 Jun;53(3):171-7. PMID:22770105 
26. McLaurin KK, Hall CB, Jackson EA, Owens OV, Mahadevia PJ. Persistence of morbidity and cost differences between latepreterm and term infants during the first year of life. Pediatrics. 2009 Feb;123(2):653-9. PMID:19171634

27. Petrini JR, Dias T, McCormick MC, Massolo ML, Green NS, Escobar GJ. Increased risk of adverse neurological development for late preterm infants. J Pediatr. 2009 Feb;154(2):169-76. PMID:19081113

28. Abu-Salah O. Unfavourable outcomes associated with late preterm birth: observations from Jordan. J Pak Med Assoc. 2011 Aug;61(8):769-72. PMID:22355999

29. Mohammad K, Abu Dalou A, Kassab M, Gamble J, Creedy DK Prevalence and factors associated with the occurrence of preterm birth in Irbid governorate of Jordan: a retrospective study. Int J Nurs Pract. 2015 Oct;21(5):505-10 PMID:25213160

30. Barfield W. Neonatal intensive-care unit admission of infants with very low birth weight-19 States, 2006. MMWR Morb Mortal Wkly Rep. 2010 Nov 12;59(44):1444-7. PMID:21063275

31. Gyamfi-Bannerman C, Fuchs KM, Young OM, Hoffman MK. Nonspontaneous late preterm birth: etiology and outcomes. Am J Obstet Gynecol. 2011 Nov;205(5):456.e1-6. PMID:22035950

32. Dimitriou G, Fouzas S, Georgakis V, Vervenioti A, Papadopoulos VG, Decavalas G, et al. Determinants of morbidity in late preterm infants. Early Hum Dev. 2010 Sep;86(9):587-91. PMID:20729014

33. Phaloprakarn C, Manusirivithaya S, Boonyarittipong P. Risk score comprising maternal and obstetric factors to iden- tify late preterm infants at risk for neonatal intensive care unit admission. J Obstet Gynaecol Res. 2015 May;41(5):680-8. PMID:25420697

34. Brown HK, Speechley KN, Macnab J, Natale R, Campbell MK. Neonatal morbidity associated with late preterm and early term birth: the roles of gestational age and biological determinants of preterm birth. Int J Epidemiol. 2014 Jun;43(3):802-14. PMID:24374829

35. Yee W, Amin H, Wood S. Elective cesarean delivery, neonatal intensive care unit admission, and neonatal respiratory distress. Obstet Gynecol. 2008 Apr;111(4):823-8. PMID:18378740

36. Crane JM, Murphy P, Burrage L, Hutchens D. Maternal and perinatal outcomes of extreme obesity in pregnancy. J Obst Gynaecol Can. 2013 Jul;35(7):606-11. PMID:23876637

37. Tita AT, Landon MB, Spong CY, Lai Y, Leveno KJ, Varner MW, et al.; Eunice Kennedy Shriver NICHD Maternal-Fetal Medicine Units Network. Timing of elective repeat cesarean delivery at term and neonatal outcomes. N Engl J Med. 2009 Jan 8;360(2):111-20. PMID:19129525

38. Dabbas $\mathrm{M}, \mathrm{Al}-$ Sumadi A. Cesarean section rate: much room for reduction. Clin Exp Obstet Gynecol. 2007;34(3):146-8. PMID:17937087

39. Runmei M, Terence T L, Yonghu S, Hong X, Yuqin T, Bailuan L, et al. Practice audits to reduce caesareans in a tertiary referral hospital in south-western China. Bull World Health Organ. 2012 Jul 1;90(7):488-94. PMID:22807594z 